\title{
Safety culture assessment: a tool for improving patient safety in healthcare organizations
}

\author{
V F Nieva, J Sorra
}

Qual Saf Health Care 2003;12(Suppl II):ii17-ii23

Increasingly, healthcare organizations are becoming aware of the importance of transforming organizational culture in order to improve patient safety. Growing interest in safety culture has been accompanied by the need for assessment tools focused on the cultural aspects of patient safety improvement efforts. This paper discusses the use of safety culture assessment as a tool for improving patient safety. It describes the characteristics of culture assessment tools presently available and discusses their current and potential uses, including brief examples from healthcare organizations that have undertaken such assessments. The paper also highlights critical processes that healthcare organizations need to consider when deciding to use these tools.

See end of article for authors' affiliations .....................

Correspondence to: Dr V F Nieva, Vice President, Westat, 1650 Research Blvd, Rockville, MD 20850, USA; veronicanieva@ westat.com
A ccording to the Institute of Medicine, "the biggest challenge to moving toward a safer health system is changing the culture from one of blaming individuals for errors to one in which errors are treated not as personal failures, but as opportunities to improve the system and prevent harm." ${ }^{1}$

Promoting a culture of safety has become one of the pillars of the patient safety movement. In recent years there has been increasing understanding within the healthcare industry that various factors-such as the emphasis on production, efficiency and cost controls ${ }^{2}$, organizational and individual inability to acknowledge fallibility, ${ }^{3}$ and professional norms for perfectionism among healthcare providers ${ }^{4}$ - combine to create a culture contradictory to the requirements of patient safety. Increasingly, the culture of the healthcare industry is regarded as a potential risk factor threatening the patients for whom it provides care.

Professional and organizational cultures in health care must undergo a transformation in the interests of promoting safer patient care. Health care must come to see itself as a high hazard industry which is inherently risky. ${ }^{5}$ It must abandon the philosophy of requiring perfect, error free performance from individuals and focus, instead, on designing systems for safety. Healthcare systems must move away from the current "blame and shame" culture that prevents acknowledgement of error and therefore obstructs any possibility of learning from error. Safety improvement requires that healthcare systems have ready access to information that supports learning from experience in order to promote systems that both prevent errors and mitigate the impact of errors that occur. ${ }^{6}$ In contrast to a "pathological culture" where failure is punished or concealed and people refuse to acknowledge that problems exist, ${ }^{7}$ a positive safety culture recognizes the inevitability of error and proactively seeks to identify latent threats.

While a variety of levers - clinical training and guidelines, information technology, organizational structures and industry regulations-are being pushed in healthcare organizations to improve patient safety, the belief is growing that an institution's ability to avoid harm will be realized only when it is able to create a culture of safety among its staff. Safety culture is a performance shaping factor that guides the many discretionary behaviors of healthcare professionals toward viewing patient safety as one of their highest priorities.

A fundamental culture change is necessary to ensure that innovations introduced to improve patient safety actually achieve their potential. For example, adverse event reporting systems will not overcome chronic underreporting problems ${ }^{3}$ within a punitive culture where acknowledgement of error is not acceptable. Analytical methods such as root cause analysis (RCA) and failure mode effects analyses (FMEA) will not succeed in uncovering latent sources of error if staff, bound by an implicit "code of silence" and a fear of challenging the institutional hierarchy, are uncomfortable with exposing weaknesses in processes for which they are responsible. Even benefits from new technologies designed to improve safety, such as computerized physician order entry, may not be realized if they are not accompanied by cultural and process changes.

Interest in safety culture assessment in healthcare organizations has grown in parallel with the increasing focus on improving safety culture. In order to transform culture it is important to first understand and confront it. Culture assessment tools provide an avenue towards such understanding. From understanding, action may emerge. This paper discusses the use of safety culture assessment as a tool for improving patient safety. It describes the characteristics of assessment tools presently available and discusses their current and potential uses, including brief examples from healthcare organizations that have undertaken such assessments. It also highlights critical processes that healthcare organizations need to consider when deciding to use these tools.

\section{SAFETY CULTURE ASSESSMENT IN HEALTHCARE ORGANIZATIONS}

The Advisory Committee on the Safety of Nuclear Installations ${ }^{8}$ provides the following definition of safety culture that can easily be adapted to the context of patient safety in health care: 
"The safety culture of an organization is the product of individual and group values, attitudes, perceptions, competencies, and patterns of behavior that determine the commitment to, and the style and proficiency of, an organization's health and safety management. Organizations with a positive safety culture are characterized by communications founded on mutual trust, by shared perceptions of the importance of safety and by confidence in the efficacy of preventive measures."

The conceptual breadth of the safety culture concept illustrated in this definition is reflected in the wide range of topics covered by safety culture assessment instrument. These instruments often assess the values, attitudes, behaviours, and norms of organization members. They may also focus on perceptions of the organizational context, such as managerial priorities, adequacy of training and resources, or policies and procedures.

An important characteristic of safety culture assessment tools is whether they take a managerial or staff perspective, or combine elements of both. Some measurement tools focus on management assessments of patient safety policies and practices in their organizations. These tools assess managerial perspectives about what they see as occurring, or needing to occur, in their organizations, as represented by formal policies and standard operating practices. These instruments are intended to provide the leadership in healthcare organizations with information about the status of official organizational practices, to generate awareness about patient safety practices, and to motivate them to take action on areas needing improvement.

An example of a management self-assessment tool focused on patient safety was developed by VHA (previously known as Voluntary Hospitals of America) in conjunction with the American Hospital Association (AHA) entitled "Strategies for Leadership: An Organizational Approach to Patient Safety". 9 The instrument is intended to be used by multidisciplinary teams, including both direct care providers and middle and top managers in hospital settings. Items are organized according to key safety aspects such as patient safety as a leadership priority, promoting a non-punitive culture for sharing information, fostering teamwork, routinely assessing the risk of errors and adverse events, and involving patients and families in care delivery. For each key aspect, managers are asked to respond to statements that describe pertinent activities using a 5 point scale to indicate the extent to which the activity has been implemented throughout the organization (from "there has been no discussion around this activity" to "this activity is fully implemented throughout the organization"). Examples of statements used to assess one of the key aspects in this assessment tool, "Promotion of a nonpunitive culture", are shown in box 1 .

Other safety culture assessment tools focus on staff perceptions and attitudes. Rather than eliciting the views of senior managers, these instruments focus on perceptions of what occurs in the daily life of the organization from the perspective of direct patient care providers and other staff who have an impact on patient safety. These tools belong to a long tradition of quantitative organizational culture and climate assessments in health care $^{10}$ and safety culture studies in a variety of high risk industries such as offshore oil drilling, air traffic control, aircraft carrier maintenance, and manufacturing. ${ }^{11}$

These staff based assessments are structured self-report surveys that elicit perceptions of the working environment from the perspective of staff at the "sharp end" of healthcare delivery in various settings (for example, emergency rooms, intensive care units, hospitals, nursing homes, or ambulatory care clinics). Typically, healthcare staff are asked to respond to a list of descriptive statements that are designed to operationalize various safety culture domains. Respondents
Box 1 Examples of management items to measure promotion of a non-punitive culture

- The organization has a non-punitive policy to address patient adverse events including medical staff and organization employees.

- The activity of legal counsel is aligned with the patient safety agenda to ensure consumer, public and legal accountability, while concurrently protecting the organization.

- Leadership encourages and rewards recognition and reporting of adverse events and near misses.

indicate their agreement (for example, from "strongly disagree" to "strongly agree") or the frequency with which events described occur (for example, from "never" to "always"). Examples of items in these staff based assessment tools are shown in box $2 .^{12}$

These instruments derive numerical scores that indicate the type of culture characterizing the organization, such as a group oriented or hierarchical culture. ${ }^{13}$ Scores may also be used to indicate the organization's standing on multiple culture domains such as openness of communication, teamwork, or perceptions of event reporting. The scores can be calculated at different levels of aggregation-the organization as a whole, organizational units (departments, clinical areas, hospital wings or floors), or different professional groups (physicians, nurses or laboratory staff).

Much research is currently underway to develop and use safety culture assessment tools. For example, in 2000 the US Veterans Health Administration launched a large scale effort to measure prevailing beliefs and behavior surrounding safety and errors in all VA hospitals. ${ }^{14}$ At the University of Texas patient safety researchers have developed a number of related assessment instruments adapted from aviation crew resource management measures to study culture within various hospital units. ${ }^{15}$ Between 2000 and 2003 the Agency for Healthcare Research and Quality (AHRQ) funded over 100 patient safety research grants and contracts. A number of these research projects use or have developed safety culture and organizational culture assessment tools.

\section{USES OF SAFETY CULTURE ASSESSMENT IN HEALTHCARE ORGANIZATIONS}

Implementing a safety culture assessment involves the commitment of staff time and resources. Why do healthcare organizations decide to assess safety culture? How are the data used? The answers to these questions can be good predictors of the extent to which culture data eventually contribute to real patient safety improvement in an institution.

Healthcare organizations may conduct safety culture assessments for a variety of reasons, but they are not

Box 2 Examples of items in staff based culture assessment instruments

- When a mistake is discovered, we try to figure out what problems in the work process led to the mistake.

- Supervisors and employees discuss how to handle incidents involving error.

- Employees feel like event reports are held against them. 
mutually exclusive and, indeed, can often occur in combination. Culture assessments can be used to: (1) diagnose safety culture to identify areas for improvement and raise awareness about patient safety; (2) evaluate patient safety interventions or programs and track change over time; (3) conduct internal and external benchmarking; and (4) fulfil directives or regulatory requirements.

\section{Diagnosing safety culture and raising awareness}

A safety culture assessment provides an organization with a basic understanding of the safety related perceptions and attitudes of its managers and staff. Safety culture measures can be used as diagnostic tools to identify areas for improvement. Because there are many potential starting points for improvement efforts, a safety culture assessment can help an organization to identify areas that are considered more problematic than others. Cultural issues that are identified as problematic can provide material for further analysis of underlying "root causes" and for generating improvement ideas from staff directly involved in the issues.

Safety culture assessment can also launch an organization's patient safety program. Assessing patient safety culture has a corollary effect, intended or not, of raising awareness levels about the role of culture in promoting a safer patient environment. Assessments communicate what is important to an organization, what are desirable end states, and what factors are viewed as leading to those end states. ${ }^{16}{ }^{17}$ Safety culture assessments can function as symbolic communications that focus attention on cultural priorities and establish a common vocabulary and set of goals to rally behind. In this way, assessment in itself may be regarded as a patient safety intervention.

\section{Evaluating patient safety interventions or programs and tracking change over time}

Changes in safety culture can be used as evidence of the effectiveness of patient safety programs and interventions. In this context, culture change is regarded as an "outcome measure", usually in conjunction with more direct measures of patient safety such as error rates and clinical outcomes. Safety culture assessments provide a way of tracking progress in cultural transformation over time. Baseline measures of culture can be taken before a patient safety intervention is implemented, with follow up measures after the intervention is underway. The scale of these assessments and the frequency with which they are conducted will differ depending on the program or intervention under evaluation.

Safety culture change is currently being tracked as part of several large scale patient safety programs. Baseline culture measures have been taken in the US Veterans Health Administration $^{14}$ and periodic assessments are planned in the future as part of an ambitious patient safety program that includes a patient safety reporting and analysis system, technology usability assessments, and methodologies for prioritizing safety related actions. ${ }^{18}$ Johns Hopkins Hospital is using safety culture measures, among others, to assess the impact of interventions implemented within their comprehensive patient safety program-including patient safety education, an active multidisciplinary safety committee that reviews the hospital's programs, policies and procedures, and executive walk arounds. ${ }^{19}$

In organizations with ongoing patient safety improvement programs, periodic safety culture measurements can be used to refine changes in repeated Plan-Do-Study-Act (PDSA) cycles. $^{20}{ }^{21}$ A continuing measurement effort can be used as part of a formative evaluation effort that is an integral part of a safety improvement program. Optimally, safety culture assessments would become part of an organizational learning and continuous improvement process.

\section{Conducting internal and external benchmarking}

Theoretically, safety culture assessments can be used to compare units within one organization or to examine differences across different organizations or systems. Such benchmarking comparisons have grown in popularity in the quality improvement and consumer empowerment movements in various settings, including health care. Internal benchmarking can be conducted with relative ease when a culture assessment tool is used across the various departments and clinical areas of a healthcare organization. Often, data are provided to unit managers, comparing their specific information with data from the entire organization.

External benchmarking is technically possible when a common assessment tool is used across many organizations. Benchmarked data can be used by healthcare consumers choosing healthcare delivery organizations, and by the organizations' quality improvement and competitor analysis efforts. For example, in the US the National Committee for Quality Assurance (NCQA) publishes the Quality Compass which provides national, regional, and individual health plan data on performance (Health Plan Employer Data and Information Set: HEDIS $)^{22}$ and customer satisfaction (Consumer Assessments of Health Plans: CAHPS). ${ }^{23}$ Report cards about hospitals provide consumers with comparative data on customer satisfaction and various aspects of patient care. In Canada, for example, the Ontario Hospital Association and the government of Ontario collaborate to produce "Hospital Report 2002: Acute Care" ${ }^{24}$ which presents comparative data for 92 acute care hospitals.

Clearly, healthcare organizations are interested in the potential for benchmarking as they decide to undertake safety culture assessments. However, organizational culture assessments are in the early stages of development; whether the data can actually be consolidated and standardized to the point of being useful for external benchmarking remains to be seen.

\section{Fulfilling directives or regulatory requirements}

Healthcare organizations are beginning to be motivated to undertake safety culture assessments to fulfil directives passed down through membership in a larger healthcare system, consortium, or through payer groups who have a stake in effective and safe healthcare delivery. Other healthcare organizations are undertaking safety culture assessments to provide regulatory agencies with evidence of their patient safety activities. Some hospitals in the US have expressed interest in safety culture assessment as one way of fulfilling standards issued by the Joint Commission on Accreditation of Health Care Organizations (JCAHO). While safety culture assessment is not a specific mandate, JCAHO does require that hospitals collect data to monitor performance, including data on staff opinions and needs, staff's willingness to report medical/health care errors, perceptions of risks to patients, and suggestions for improving patient safety. ${ }^{25}$

\section{CRITICAL PROCESSES IN SAFETY CULTURE ASSESSMENT}

To achieve maximal benefit from conducting a safety culture assessment, healthcare organizations must attend to several critical processes-from involving key stakeholders to planning safety improvements based on the data. We have selected these critical processes because they are potential stumbling blocks for organizations attempting to use safety culture assessment as a tool for patient safety improvement. 


\section{Involvement of key stakeholders}

The decision to conduct a safety culture assessment effort and subsequent action planning must involve stakeholders whose support is required, who have an interest in the results, or who will need to be involved in the data collection process. While specialized staff such as quality improvement professionals, risk managers, or patient safety officers of a healthcare organization may be in charge of the logistics of safety culture assessment, communication with senior and middle managers as well as employees is essential to clarify the purposes of the initiative and to establish commitment to the effort.

Calling for leadership involvement in organizational assessment efforts may appear to be so obvious as to be an unnecessary platitude, yet instances where this step is overlooked are not uncommon. For example, in one regional consortium of hospitals, plans for a safety culture assessment effort were derailed when senior management and other key stakeholders who were not involved in the initial planning of the effort voiced major objections to the issues covered in the tool that was selected. The process had to be restarted by working with the stakeholders to redevelop a rationale that addressed their specific patient safety concerns, outlining how the data would be used, and selecting an appropriate tool to accomplish their objectives.

The involvement of senior management such as the CEO, President, COO, and even board members is especially critical because they are ultimately responsible for policy and strategic decisions and they will be expected to do something about the results. ${ }^{16}$ In addition, senior management controls the resources necessary to address areas identified as needing attention. The benefits of involving senior management were exemplified at a large university hospital that involved its CEO in a patient safety rounds program where senior managers periodically visited a hospital unit to speak with staff firsthand about patient safety issues in the unit. After conducting the rounds, the CEO took personal responsibility for making sure that every problem that was raised by unit staff was resolved in a timely manner.

Clinical staff, and physicians in particular, are also important stakeholders. Lessons can be learned from the experience of the Continuous Quality Improvement (CQI) movement in health care. A review of CQI over the past decade $^{26}$ concluded that quality improvement efforts have made limited inroads into the clinical side of healthcare organizations due to failures to effectively include physicians and their patient care issues in improvement initiatives.

Obtaining stakeholder support can be daunting in a healthcare organization. In large healthcare systems it is often necessary to obtain support from multiple authority structures and levels-senior management; medical and nursing hierarchies; human resources; departmental units; and unions, where these exist. Some settings may also require approval from a hospital or university's internal review board (IRB) to collect data for a culture assessment. Planners of culture assessment efforts must include considerable time-often many months-to develop the collaborations necessary to involve the large variety of stakeholders and institutional gatekeepers whose support is needed. Moreover, these stakeholders are critical to the implementation of any organizational or process changes that are generated from the assessment results.

\section{Selecting a suitable safety culture assessment tool}

Once the rationale and objectives for a safety culture assessment have been clarified and all key stakeholders have been consulted, a safety culture assessment tool must be selected or developed. We recommend that healthcare organizations first examine the suitability of existing tools to their needs before embarking on an effort to develop a new tool. Criteria for suitability include: (1) the domains of culture that are assessed; (2) the types of staff who are expected to complete the tool; (3) the settings for which the tool was developed; and (4) the availability of reliability and validity evidence about the tool.

It is important to select a tool that best suits the purposes for which the data will be used and covers the aspects of culture that are of interest to the organization. If the goal is to obtain a summary view of the status of patient safety culture, an instrument that covers a few major safety topics might suffice. If the purpose is more diagnostic with the intent of identifying areas that may present high risks for patient harm, a tool that covers a broader range of safety culture areas would offer more value. To evaluate the effects of a specific patient safety intervention it is important to choose a tool that measures the specific cultural domains that will be affected.

The intended source of information for the tools-senior managers, specific types of staff such as nurses, pharmacists, or physicians, or all staff types and levels-should also be checked for suitability. Tools designed for senior managers may address issues about which other staff are typically uninformed, or elicit information specifically geared toward a management perspective. Similarly, tools designed for nurses may not address safety culture issues that reflect the concerns of physicians or administrative managers. Safety culture assessment tools are also typically targeted for specific settings. For example, some tools may focus on safety culture issues specific to hospitals while others may focus on pharmacies, ambulatory facilities, nursing homes, or intensive care units. Modification may be required when adopting a tool for a setting other than the one for which it was intended.

Information about the quality of culture assessment tools is currently difficult to find. Evidence on instrument reliability is lacking for many, and validity evidence is even more elusive. Like other patient safety improvement tools, there is limited evidence establishing a linkage between positive safety culture and positive clinical outcomes or medical error reduction. However, some studies have shown linkages between staff perceptions of culture and outcomes such as quality of care and lower risk adjusted length of stay. ${ }^{27}{ }^{28} \mathrm{~A}$ strong safety climate has also been found to be associated with compliance with safety work practices among nurses. ${ }^{29}$ As more safety culture assessments are done, more validity evidence related to culture assessment is expected.

For healthcare organizations the search for an existing safety culture assessment tool that can meet all their needs can be challenging. Although a number of tools have been developed, many are not readily accessible. Some safety culture tools are proprietary and are only available for a fee. Published research studies that use safety culture assessment tools typically do not include the full instrument; copies must be requested through the primary author. Unpublished tools can be even more difficult to locate.

Recent reviews of quantitative measures of safety culture ${ }^{11}$ and organizational culture in health care $^{10}$ provide good information about published culture assessment tools. These reviews outline the dimensions assessed, the settings in which they have been administered, the number of items, and information about their reliability and validity. However, these reviews do not include the many proprietary and unpublished tools that are available or that have recently been developed and are currently being used in healthcare organizations. Ideally, it would be very useful to have an inventory that lists both published and unpublished safety culture assessment tools that have been developed, including information on their technical specifications, usage, and 
contact information to obtain review copies. For now, however, the process of locating safety culture assessment tools to consider using will require effort and time.

\section{Using effective data collection procedures}

Collecting safety culture assessment data typically involves the use of survey administration methods. While numerous texts provide guidelines on classic survey methodologies and their application to organizational settings-for example, sampling, advance communication, follow up to maximize response rates, preventing bias in data, ${ }^{1730}$ it is not uncommon for these procedures to be overlooked by staff conducting assessments in healthcare organizations.

When procedures to collect assessment data are not well designed, the quantity, quality and generalizability of the data are likely to be negatively affected. Healthcare organizations risk obtaining assessment data that, in the end, may prove to be unusable. Response rates frequently suffer due to inadequate preparation. In one extreme case in an urban community hospital, only one staff member completed the culture assessment over a two day period. Staff were asked to go to a designated room to complete the assessment, but inadequate advance notification and staff concerns about data confidentiality were thought to have led to the lack of response. Sometimes the use of new technologies for data collection that are successful in some settings may be ill advised in healthcare organizations. For example, a number of healthcare researchers have been unable to achieve adequate responses using web based assessment tools due to the limited access of hospital employees to computers with online connections.

Procedures that result in inaccurate or biased data may be even more serious because they are harder to detect. For example, one national healthcare system instructed some of its member hospitals to have staff complete a safety culture assessment tool after viewing a videotape promoting patient safety. It is likely that staff responses to the assessment were affected by the priming effect of the video. In addition, each hospital was instructed to obtain at least 50 completed surveys but, since no guidance was provided on sampling procedures, it is not possible to determine the representativeness of the data.

Healthcare organizations collecting their own assessment data should become knowledgeable about survey administration procedures to prevent scenarios like these. Organizations should not underestimate the knowledge and level of effort that is required not only to collect the data, but to analyze and synthesize the results. Failure to attend to these processes can seriously affect the outcomes of an assessment effort.

\section{Implementing action planning and initiating change}

If a safety culture assessment reveals a punitive culture that suppresses adverse event reporting, how does an organization move from these data to usable knowledge, and from knowledge to sustainable change? The effectiveness of safety culture data as a tool for patient safety improvement requires processes for developing a shared organizational understanding of the underlying meanings and causes of the data, and for identifying the range of potential actions relevant to those interpretations. Rather than viewing the assessment results as an end point, the information should be considered the starting point from which action and patient safety changes emerge.

Practitioners in data based cultural transformation, organizational change, and CQI ${ }^{17}{ }^{21}{ }^{31-33}$ discuss the importance of using a systematic process involving data feedback, problem solving, action planning, and monitoring to facilitate the progression from data to action. Results are typically provided to top managers after a culture assessment, but one of the most common complaints from employees who participate in these assessments is the lack of feedback about the results and any subsequent improvement actions. If safety culture assessments are to lead to culture change, feedback should be provided to all who contribute to the assessment. Results can be presented by organization or facility, by unit or team, by staff categories, or other groupings relevant to the purposes of the assessment. In this way, assessment data can be used for localized patient safety improvement efforts at various levels and sections of the organization.

For greater impact, feedback can be combined with action planning sessions. These sessions have been shown to be most effective when they are conducted by trained line managers rather than top management, external experts, or specialized staff. ${ }^{31}$ In healthcare organizations clinical staff, departmental managers, and supervisors must be involved in leading feedback discussions, not just delegating these functions to specialized staff in the quality improvement, patient safety, or risk management departments. The fruitfulness of the data utilization process can rest heavily on the skill of the session leaders. In the hands of "naïve" facilitators, sessions can easily deteriorate into unproductive defensiveness and negativism. Because facilitation and action planning require specialized skills, healthcare managers and clinicians should be provided with specific training and action planning aids to enable them to be comfortable and effective in these roles.

Feedback and action planning sessions are typically conducted in groups that have been assembled for this specific purpose. These groups are designed in different ways, depending on the nature of the organization and its goals. Feedback and action planning sessions must be designed with care, bringing together multidisciplinary groups while recognizing the complexities of healthcare organizations and their dual clinical and administrative authority structures.

Assessment data are likely to point to many different areas of culture that could be improved, accompanied by different interpretations about potential actions that could be taken in each area. Incremental changes can be implemented and tested on a small scale, changing one process or practice at a time, in only particular units of the organization, or over a short trial period..$^{21}$ Improvements in aviation safety over the years have relied on the widespread implementation of hundreds of small changes in procedures, equipment, training, and organization that aggregated to establish effective practices and a strong safety culture. ${ }^{34}$ In patient safety, as in aviation, there is no one "silver bullet".

\section{CONCLUSIONS}

Safety culture assessments are new tools in the patient safety improvement arsenal. These tools can be used to measure organizational conditions that lead to adverse events and patient harm, and for developing and evaluating safety improvement interventions in healthcare organizations. They provide a metric by which the implicit shared understandings about "the way we do things around here" can be made visible and available as input for change.

Healthcare organizations are only beginning to work with culture assessment tools and with the concept of safety culture itself. There is more to learn regarding creating and sustaining culture change in health care and the tools that might be used in these transformation efforts. Much remains to be discovered on how to use culture data in combination with other sources of information about patient safety improvement needs in different organizational contexts. Like other new patient safety improvement tools, there is room for further development on several fronts: accumulating evidence about the validity of these tools, learning how to initiate and sustain safety culture change, and discovering 


\section{Pointers for future research}

- More evidence is needed about the validity of safety culture assessment tools.

- We need to learn how to use assessment data to initiate and sustain safety culture change.

- Culture assessment data must be combined with other patient safety information in making decisions about ways to improve patient safety.

how to use culture data in combination with other sources of information about patient safety.

As healthcare organizations experiment with efforts to improve patient safety including the use of culture assessment tools, understanding of the usefulness of the cultural perspective will grow as well. While some evidence is available on the validity of some culture tools, this evidence base must be expanded. The links between various culture measures and outcomes such as quality of care and patient safety must be demonstrated further. Also, the industry needs more examples from organizations that have assessed culture and successfully used the data to initiate change.

Prescriptive guidance on how to create cultural change is still limited, although there is emerging consensus on some of the cultural attributes that contribute to patient safety such as teamwork, leadership support, and communication. There are likely to be many roads to achieving a positive safety culture. The equifinality concept in systems theory, ${ }^{35}$ which is applicable to our understanding of safety culture, asserts that the final state of a system may be reached from different initial conditions and in different ways. Thus, an organization with a particular set of cultural attributes may be successful in achieving patient safety, while another organization with a different set of cultural attributes can also potentially achieve the same levels of success.

While this paper clearly advocates that quantitative measures of safety culture offer promise as tools for patient safety improvement, we recognize the limitations of this

\section{Key messages}

- Safety culture assessments are useful tools for measuring organizational conditions that lead to adverse events and patient harm in healthcare organizations.

- Safety culture assessments can have multiple purposes:

- diagnosis of safety culture and raising awareness;

- evaluation of patient safety interventions and tracking change over time;

- internal and external benchmarking;

- fulfilment of regulatory or other requirements.

- The usefulness of safety culture assessment data depends on:

- involving key stakeholders;

- selecting a suitable safety culture assessment tool;

- using effective data collection procedures;

- implementing action planning and initiating change.

- Safety culture assessment should be viewed as the starting point from which action planning begins and patient safety changes emerge. approach. The deeper aspects of culture in terms of underlying values, beliefs, and norms within an organization may be inadequately captured with self-report quantitative instruments. Individuals embedded in a culture are often unconscious of and inarticulate about the culture that surrounds them. Quantitative culture data should therefore be supplemented with other sources of information about patient safety such as qualitative information from staff interviews and focus groups, or procedural safety checklists used in traditional safety audits. Since patient safety tools are still developing, there is more to learn about how data obtained from different tools are related and how to combine these data to get the most comprehensive view of patient safety.

\section{Authors' affiliations}

V F Nieva, J Sorra, Westat, Research Blvd, Rockville, MD 20850, USA

The contents of this paper are the work of the author(s) and do not necessarily represent the opinions, recommendations, or practices of Westat.

\section{REFERENCES}

1 Institute of Medicine. Crossing the quality chasm: a new health system for the 21 st century. Washington, DC: National Academy Press, 2001

2 Gaba DM, Howard SK, Jump B. Production pressure in the work environment. California anesthesiologists' attitudes and experiences. Anesthesiology 1994;81:488-500.

3 Leape LL. Error in medicine. JAMA 1994:272:1851-7.

4 Krizek TJ. Surgical error: ethical issues of adverse events. Arch Surg 2000;135:1359-66.

5 Leape LL, Woods DD, Hatlie MJ, et al. Promoting patient safety by preventing medical error. JAMA 1998;280:1444-7.

6 Reason JT. Organizational accidents: the management of human and organizational factors in hazardous technologies. Cambridge: Cambridge University Press, 1997.

7 Westrum R. Cultures with requisite imagination. In: Wise JA, Hopkin VD, Stager $P$, eds. Verification and validation of complex systems: human factors issues. 1992:401-16.

8 Health and Safety Commission (HSC). Organizing for safety: Third report of the human factors study group of ACSNI. Sudbury: HSE Books, 1993.

9 VHA/American Hospital Association (AHA). Strategies for leadership: an organizational approach to patient safety. American Hospital Association, Item \# 166925, 2001.

10 Scott T, Mannion R, Davies H, et al. Methods. Tthe quantitative measurement of organizational culture in health care: a review of the available instruments. Health Serv Res 2003;38:923-45.

11 Guldenmund F. The nature of safety culture: a review of theory and research. Saf Sci 2000;34:215-57.

12 Sorra J, Nieva V, Schreiber G, et al. Safety culture and event reporting in hospital transfusion services. American Association of Blood Banks (AABB) Annual Meeting, Orlando, FL, 2002.

13 Quinn RE, Kimberly JR. Paradox, planning, and perseverance: guidelines for managerial practice. Homewood, IL: Dow Jones-Irwin, 1984.

14 McKnight S, Lee C. Patient safety attitudes. Paper presented at the Summit on Effective Practices to Improve Patient Safety, Washington DC, 2001.

15 Sexton JB, Thomas EJ, Helmreich RL. Error, stress, and teamwork in medicine and aviation: cross sectional surveys. BMJ 2000;320:745-9.

16 Church A, Waclawski J. Designing and using organizational surveys: a sevenstep process. San Francisco: Jossey-Bass, 1998.

17 Kraut Al, ed. Organizational surveys: tools for assessment and change. San Francisco: Jossey Bass, 1996.

18 Bagian JP, Lee C, Gosbee J, et al. Developing and deploying a patient safety program in a large health care delivery system: you can't fix what you don't know about. Jt Comm J Qual Improv 2001 ;27:522-32.

19 Anon. Improving patient safety: from rhetoric to reality. Conference jointly sponsored by Johns Hopkins University School of Medicine, The Health Research and Educational Trust and Partners HealthCare System's Brigham and Women's Hospital; co-sponsored by the Institute for Johns Hopkins Nursing, May 2003.

20 Berwick DM. A primer on leading the improvement of systems. BMJ 1996;312:619-22.

21 Langley G, Nolan K, Norman C, et al. The improvement guide. San Francisco: Jossey-Bass, 1996.

22 National Committee for Quality Assurance (NCQA). Health plan data and information set (HEDIS) 2004. Volume 3. Item \# 10285-100-04. Washington, DC: NCQA, 2003.

23 Agency for Healthcare Research and Quality (AHRQ). Consumer assessments of health plans (CAHPS) survey and reporting Kit 2002. Version 3.0. Rockville, MD: AHRQ, 2002.

24 Ontario Hospital Association. Highlights of hospital report 2002: Acute care. Ontario Hospital Association, 2002. 
25 Joint Commission on Accreditation of Healthcare Organizations (JCAHO) Comprehensive accreditation manual for hospitals. Chapter on improving organization performance. Standard PI.3.1 and the intent for PI.3.1-The organization collects data to monitor its performance. Oakbrook Terrace, IL: JCAHO, 2003.

26 Blumenthal D, Kilo CM. A report card on continuous quality improvement. Milbank Q 1998;76:625-48:511511.

27 Shortell SM, Rousseau DM, Gillies RR, et al. Organizational assessment in intensive care units (ICUs): construct development, reliability, and validity of the ICU nurse-physician questionnaire. Med Care 1991;29:709-26.

28 Shortell SM, Zimmerman JE, Rousseau DM, et al. The performance of intensive care units: does good management make a difference? Med Care 1994;32:508-25

29 Grosch JW, Gershon RR, Murphy LR, et al. Safety climate dimensions associated with occupational exposure to blood-borne pathogens in nurses. Am J Ind Med 1999; Suppl 1:122-4.
30 Dillman DA. Mail and internet surveys: the tailored design method. 2nd ed. New York: Wiley, 2000.

31 Hinrichs J. Feedback, action planning and follow-through. In: Kraut A, ed. Organizational surveys: tools for assessment and change. San Francisco: Jossey Bass, 1996:255-79.

32 Wagner $D$, Spencer J. The role of surveys in transforming culture: data, knowledge, action. In: Kraut A, ed. Organizational surveys: tools for assessment and change. San Francisco: Jossey Bass, 1996:67-87.

33 Lawler E, Nadler D, Cammann C. Organizational assessment: perspectives on the measurement of organizational behavior and the quality of work life. New York: John Wlley, 1980.

34 Leape LL, Berwick DM, Bates DW. What practices will most improve safety? Evidence-based medicine meets patient safety. JAMA 2002;288:501-7.

35 Miller J. Living systems. New York: McGraw-Hill, 1978. 